\title{
ArcheoSciences
}

Revue d'archéométrie

\section{Les blocs carbonisés de millet commun de l'habitat protohistorique de Villiers-sur-Seine « Le Gros Buisson » (Seine-et-Marne) : peut-on parler de préparation alimentaire?}

Analysis of charred lumps of broomcorn millet found on the Late Bronze Age settlement of Villiers-sur-Seine " Le Gros Buisson » (Seine-et-Marne

department). Could they result from food processing?

\section{Françoise Toulemonde}

\section{(2) OpenEdition}

\section{Journals}

Édition électronique

URL : https://journals.openedition.org/archeosciences/4192

DOI : $10.4000 /$ archeosciences.4192

ISBN : 978-2-7535-3691-3

ISSN : 2104-3728

\section{Éditeur}

Presses universitaires de Rennes

\section{Édition imprimée}

Date de publication : 30 novembre 2014

Pagination : 109-118

ISBN : 978-2-7535-3689-0

ISSN : $1960-1360$

\section{Référence électronique}

Françoise Toulemonde, "Les blocs carbonisés de millet commun de l'habitat protohistorique de Villiers-sur-Seine «Le Gros Buisson » (Seine-et-Marne) : peut-on parler de préparation alimentaire? », ArcheoSciences [En ligne], 38 | 2014, mis en ligne le 30 novembre 2016, consulté le 28 janvier 2022. URL : http://journals.openedition.org/archeosciences/4192 ; DOI : https://doi.org/10.4000/ archeosciences.4192 


\title{
Les blocs carbonisés de millet commun de l'habitat protohistorique de Villiers-sur-Seine «Le Gros Buisson » (Seine-et-Marne) : peut-on parler de préparation alimentaire?
}

\author{
Analysis of Charred Lumps of Broomcorn Millet Found on the Late Bronze Age \\ Settlement of Villiers-sur-Seine "Le Gros Buisson" (Seine-et-Marne department) \\ Could they Result from Food Processing?
}

\author{
FranÇOISE TOULEMONDE
}

\begin{abstract}
Résumé : Des conglomérats carbonisés de millet commun (Panicum miliaceum) ont été retrouvés en grand nombre sur l'habitat fortifié francilien de Villiers-sur-Seine "Le Gros Buisson ", daté de la transition Bronze/Fer. Pour comprendre la nature de ces restes végétaux qui ne proviennent pas d'un incendie de stocks, une enquête a été menée à l'aide d'exemples archéologiques, d'études ethnographiques et ethnohistoriques et d'expérimentations sur du millet actuel. Les conclusions de cette enquête ne sont pas en faveur d'une préparation alimentaire mais plutôt d'un traitement thermique des grains, très souvent attesté dans les exemples ethnographiques. Il permet de faciliter le décorticage ou de mieux conserver les grains. Si d'autres interprétations ne sont pas exclues, elles sortent du domaine strict de l'alimentation végétale et peuvent difficilement faire l'objet d'une démonstration.
\end{abstract}

\begin{abstract}
Numerous lumps of charred kernels of broomcorn millet (Panicum miliaceum) were found on the fortified site of Villiers-sur-Seine "le Gros Buisson", located in the Ile-de-France region and dated from the Late Bronze Age/Early Iron Age. In order to understand how these lumps formed (stocks fire is not likely), researches were done within archaeobotanical studies, and ethnographical and ethnohistorical surveys. Experiments with broomcorn millet were also carried out. Conclusions do not favour ready-to-eat processed food, but more likely thermic treatments of grains, which are very often quoted in ethnographical examples. Their goal is to facilitate dehusking or to allow better grain preservation. Other interpretations cannot be excluded, but are out of the field of vegetal food processing; they cannot be demonstrated here.
\end{abstract}

Mots clés : Millet commun, Panicum miliaceum, Âge du Bronze, carpologie, préparation alimentaire, Île-de-France.

Keywords: Broomcorn millet, Panicum miliaceum, Bronze Age, archaeobotany, processed food, Ille-de-France region.

\section{INTRODUCTION}

L'habitat fortifié francilien de Villiers-sur-Seine " Le Gros Buisson ", occupé au viII siècle av. J.-C., a livré un grand nombre de conglomérats carbonisés de millet com- mun (Panicum miliaceum), dispersés sur l'ensemble du site. Il semble peu probable qu'il s'agisse de reliquats de stocks incendiés. Dès lors, que représentent ces blocs? De quelle activité de traitement des grains sont-ils le résultat? Est-ce qu'il peut s'agir d'une préparation alimentaire? Pour tenter

a UMR CNRS/MNHN 7209 "Archéozoologie, archéobotanique : sociétés, pratiques et environnements " Muséum national d'Histoire naturelle, CP56 - 55 rue Buffon, 75005 Paris. (francoise.toulemonde@mnhn.fr) 
d'approcher au plus près leur nature, une enquête est menée qui fait appel tour à tour à des précédents archéologiques, à des données ethnographiques et à des expérimentations sur la céréale actuelle.

\section{LE CONTEXTE DE DÉCOUVERTE}

\section{L'habitat protohistorique de Villiers-sur-Seine « le Gros buisson »}

Une opération d'archéologie préventive en Seine-etMarne, menée en 2005 par l'Inrap (Institut national de recherches archéologiques préventives) sous la direction de la responsable d'opération Rebecca Peake, a mis au jour, dans la vallée de la Seine, un habitat fortifié installé sur une butte graveleuse d'environ 2,3 hectares. La zone occupée dessine un triangle enserré par la Seine, un paléochenal et le plus long d'une série de fossés concentriques (figure 1). À l'intérieur, deux grands bâtiments, des fossés doublés de palissades dont l'une comporte une entrée monumentale, et des fosses en très grande quantité se distribuent l'espace. Le caractère fortifié et ostentatoire du site, la présence d'une activité de métallurgie, l'abondance et la qualité du matériel tant céramique que métallique, et la mise en évidence d'une importante activité de chasse confèrent à cet habitat un sta-

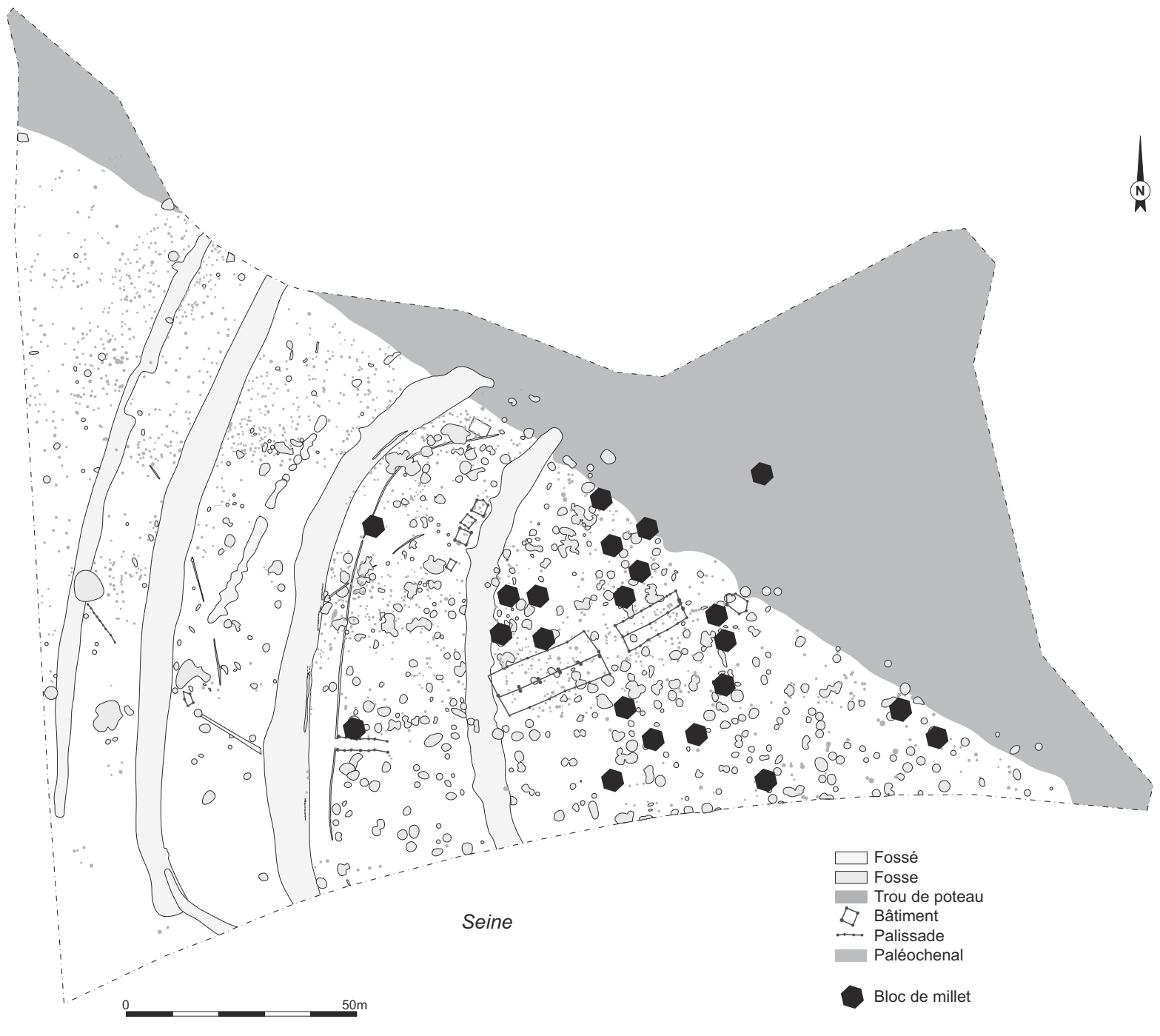

Infographie P. Pihuit (Inrap), complétée F. Toulemonde

Figure 1 : (Voir planche couleur) Plan général du site de Villiers-sur-Seine « Le Gros Buisson » avec indication de la situation des blocs de millet commun (P. Pihuit, Inrap, complétée par F. Toulemonde).

Figure 1: (See colour plate) Map of the settlement of Villiers-sur-Seine "Le Gros Buisson", with indication of the findings of lumps of broomcorn millet (P. Pihuit, Inrap, complemented by F. Toulemonde). 
tut hors du commun (Peake et al., 2009). Son occupation, datée du viII siècle av. J.-C., a duré environ cinquante ans.

\section{L'économie végétale}

En dépit de la nature très grossière du sédiment encaissant, constitué de sables et de graviers, les macro-restes végétaux ont bénéficié d'une bonne conservation en quantité et en qualité, tout comme l'ensemble du matériel retrouvé sur le site. Les restes carbonisés proviennent tous de structures de rejet, fosses ou silos en réemploi. Ils témoignent d'une agriculture très diversifiée, typique de l'âge du Bronze final. Les céréales dominantes forment une triade : orge vêtue (Hordeum vulgare), blé amidonnier (Triticum dicoccum) et millet commun (Panicum miliaceum). Cette triade est accompagnée, en moindre quantité, d'un blé nu (Triticum aestivum/durum/turgidum), d'épeautre (Triticum spelta) et de quelques spécimens d'orge nue (Hordeum vulgare var. nudum). Les légumineuses (lentille Lens culinaris, féverole Vicia faba var. minor, pois Pisum sativum et ers Vicia ervilia) sont à la fois fréquentes et abondantes. Un oléagineux, l'œillette (Papaver somniferum), clôt la liste des plantes cultivées tandis que plusieurs espèces de cueillette (gland Quercus sp., noisette Corylus avellana, prunelle Prunus spinosa, cynorhodon Rosa sp, poire ou pomme sauvage Pyrus/Malus sylvestris) attestent d'une exploitation du milieu environnant (Toulemonde, 2006 et 2010).

Outre les restes végétaux issus de prélèvements systématiques, des découvertes récurrentes d'amas de graines carbonisées et soudées entre elles ont donné lieu à des collectes ponctuelles. Ces blocs de graines sont constitués de millet commun pour plus de $90 \%$ d'entre eux.

\section{LES BLOCS DE MILLET}

Les conglomérats de millet ont été découverts en contexte détritique, dans le remplissage secondaire de fosses réparties sur l'ensemble du site, plus particulièrement sur la surface la plus intensément occupée, à l'intérieur des deux premiers fossés concentriques (figure 1). Il en a été retrouvé dans vingt-deux structures distinctes, où ils se présentaient comme des boules isolées au sein du sédiment. Aucun ne se trouvait à l'intérieur d'une céramique, ni clairement associé à l'une d'entre elles. Les volumes sont assez faibles : les plus gros blocs n'excèdent pas $90 \mathrm{~cm}^{3}$, pour un total d'environ $1500 \mathrm{~cm}^{3}$. Leur épaisseur maximale est de $3 \mathrm{~cm}$. Sur plusieurs échantillons, on observe des surfaces planes ou légèrement convexes, ainsi que des traces de courbure évoquant l'existence d'un contenant au moment de la carbo- nisation (figures 2 et 3). Les blocs compacts sont formés de grains carbonisés de millet commun non décortiqué, dont les glumelles sont fortement soudées les unes aux autres. À l'intérieur de ces enveloppes, les grains ont tendance à se détacher (figure 4). À l'exception d'un unique échantillon présentant quelques traces de tiges de céréales, les blocs renferment des semences de millet seules, non décortiquées de leurs glumelles mais parfaitement débarrassées de tout fragment de panicule ou de glume. Dans la chaîne opératoire de transformation du millet décrite par K. Lundström-Baudais (Lundström-Baudais et al., 2002), on se situe juste après les phases d'égrenage et de vannage : les grains ont été libérés de leurs glumes et débarrassés de celles-ci ainsi que des composantes de la panicule.

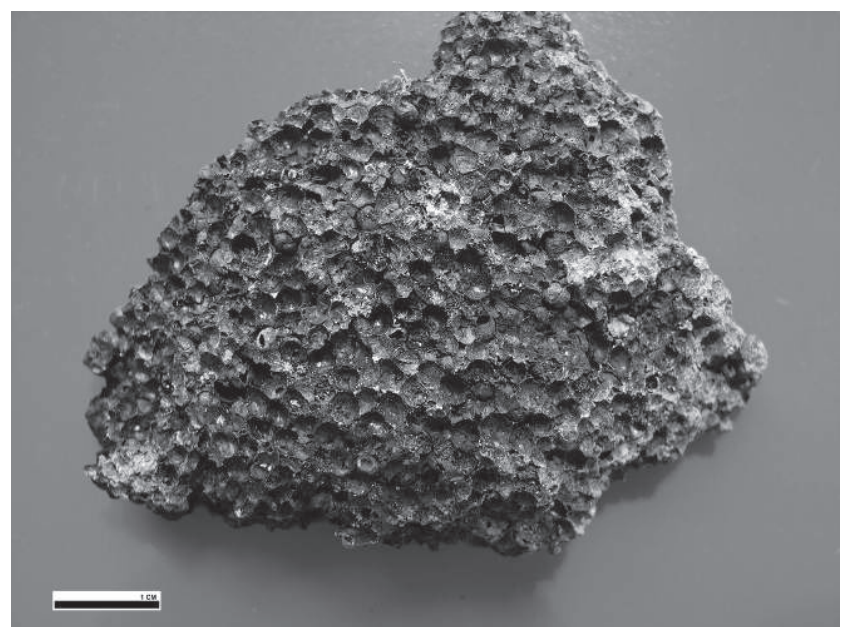

Figure 2: (Voir planche couleur) Bloc de millet commun carbonisé du site de Villiers-sur-Seine (cliché : F. Toulemonde).

Figure 2: (See colour plate) Lump of charred broomcorn millet from the settlement of Villiers-sur-Seine (picture: F. Toulemonde).

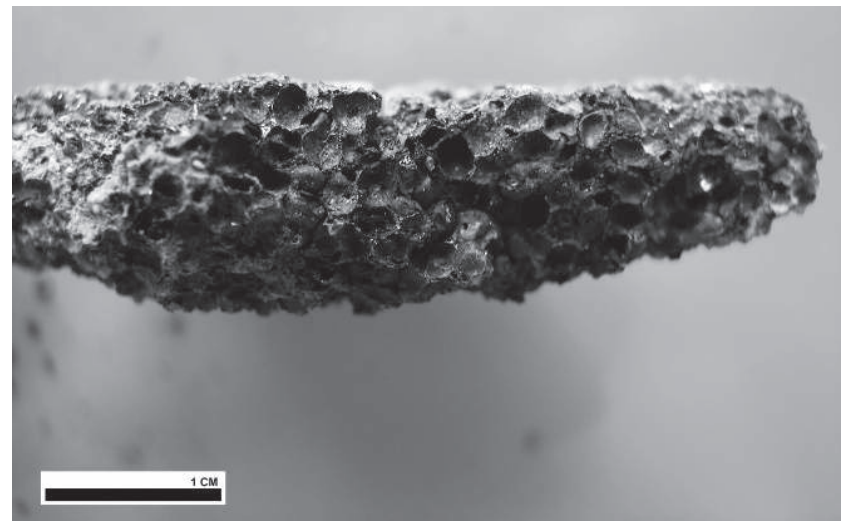

Figure 3 : Bloc de millet commun avec surface plane et courbure (cliché : F. Toulemonde).

Figure 3: Lump of charred broomcorn millet with even surface and curvature (picture: F. Toulemonde). 


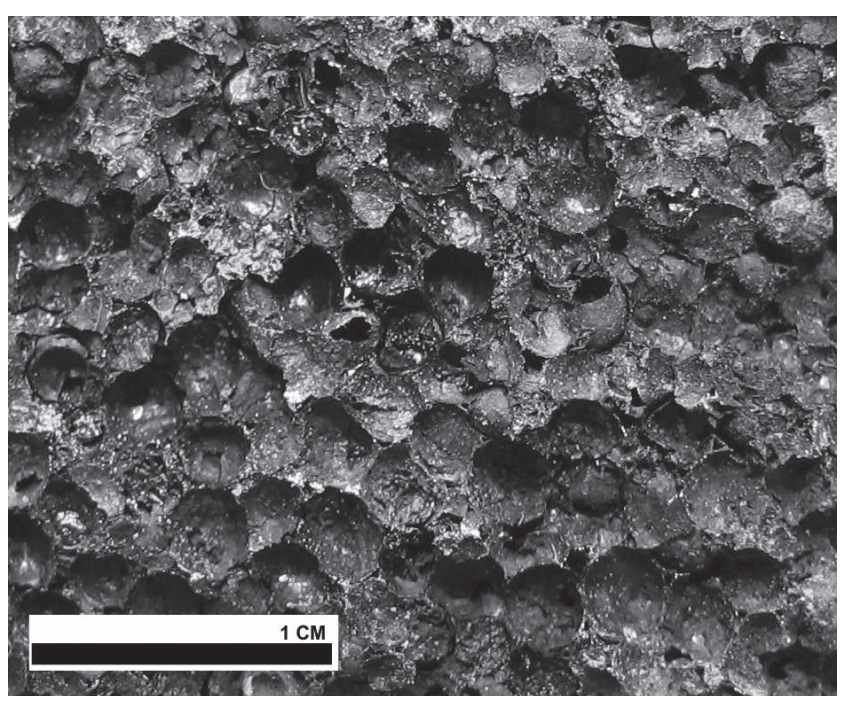

Figure 4: Vue rapprochée d'un bloc de millet commun (cliché : F. Toulemonde).

Figure 4: Close view of a lump of charred broomcorn millet (picture: F. Toulemonde).

\section{4. ÉLÉMENTS D’INTERPRÉTATION}

Quels événements ou quelles activités peuvent expliquer la carbonisation accidentelle des grains à ce stade de leur transformation? Comment les mettre en évidence?

La première source d'information et la plus immédiate pour un carpologue est bien évidemment la documentation archéologique et archéobotanique. Nous l'avons parcourue à la recherche de cas comparables et des interprétations qui en ont été données. La documentation ethnographique et ethnohistorique a ensuite été décortiquée pour retrouver les étapes de traitement du millet qui supposent un passage au feu. Puis nous avons tenté une approche expérimentale pour reproduire ces traitements et comparer le produit résultant avec le matériel archéologique.

\section{Quelques précédents archéologiques}

Les références à des conglomérats de millet commun non décortiqué sont assez nombreuses dans la littérature archéobotanique. Beaucoup proviennent d'habitats de l'âge du Bronze, ce qui n'a rien de surprenant puisque le millet est une céréale phare de cette période.

Observons quatre exemples parmi les plus significatifs dans les données publiées : l'étude de C. Bakels concernant le site de Fort-Harrouard (Eure-et-Loir) au Bronze moyen et final (Bakels, 1983), celles de L. Bouby à la Balme Gontran (Ain) et à La Baume Layrou (Gard) au Bronze final (Bouby et al., 2005), de C. Jacquat à Hauterive-Champréveyres (Suisse) au Bronze final (Jacquat, 1989) et de Ph. Marinval concernant des céramiques des époques laténienne et galloromaine retrouvées dans la Saône (Bonnamour et Marinval, 1985).

Pour ces contextes assez différents, deux types d'interprétation ont cours : l'incendie de stocks pour les deux premiers, la carbonisation d'une préparation alimentaire pour les deux autres.

Linterprétation de stocks incendiés à Fort-Harrouard est justifiée par la présence de plusieurs concentrations importantes de graines carbonisées, formées de centaines ou de milliers de semences d'orge, de blé, de millet, de légumineuses... Sur ces fouilles anciennes, le manque d'information sur les contextes limite par ailleurs les investigations et les hypothèses.

À la Balme Gontran et à la Baume Layrou, la théorie d'un incendie s'appuie sur l'existence, dans les deux grottes, de couches épaisses de grains pratiquement purs. Plusieurs espèces y sont représentées, avec des distributions plus ou moins distinctes pour chacune, ce qui permet d'envisager des stockages séparés.

À Hauterive-Champréveyres, la proposition d'une préparation alimentaire pour les conglomérats de millet est le fruit d'une comparaison avec un récipient provenant du site proche de Concise, daté du Bronze final lui aussi. Le pot en question présente sur les parois une croute composée principalement de millet non décortiqué, très proche selon l'auteur de son propre matériel.

Les céramiques trouvées dans la Saône aux alentours de Chalon-sur-Saône renferment également une croûte charbonneuse incluant des grains de millet vêtus de leurs glumelles. La présence d'un contenant et le contexte de découverte au fond d'une rivière ont conduit les auteurs à interpréter ces restes comme ceux d'une bouillie ou d'une bière. Ceux-ci auraient servi d'offrandes près de passages à gué ou d'objets de commerce transportés par voie fluviale.

Que déduire de tout ceci sur l'origine des blocs de millet de Villiers? Tout d'abord, l'hypothèse d'un incendie ne semble pas à retenir. En effet les blocs sont très disséminés sur le site alors que rien n'indique par ailleurs un incendie général. Ils ont été découverts exclusivement dans des contextes détritiques qui renfermaient également d'autres rejets domestiques. Aucune autre espèce cultivée n'a été retrouvée en quantité et en concentration suffisante pour corroborer l'idée de stocks incendiés. Enfin, les blocs euxmêmes, quoique nombreux, représentent des volumes limités : le maximum dans une même structure se monte à $310 \mathrm{~cm}^{3}$, soit l'équivalent d'un cube de $7 \mathrm{~cm}$ de côté. 
Si un incendie n'est pas à l'origine de ces restes si fréquents, ils doivent provenir d'opérations courantes et récurrentes. En dehors de la préparation alimentaire, terme vague qui peut recouvrir des produits très différents, d'autres traitements sont peut-être possibles. Et s'il s'agit vraiment d'une préparation alimentaire, un point reste à éclaircir car les blocs sont constitués de grains non décortiqués, entourés de « leurs épaisses et coriaces glumelles, donc impropres à la consommation " (Lundström-Baudais et al., 2002, p. 188). Est-il possible de se nourrir de manière régulière de millet vêtu?

\section{Données et comparatifs ethnographiques}

Pour trouver des éléments de réponse à ces différentes questions, nous avons étudié la documentation décrivant les procédés traditionnels de transformation des millets. La littérature est heureusement assez riche sur le sujet car les millets ont été cultivés en Europe jusqu'au $\mathrm{xx}^{\mathrm{e}}$ siècle et le sont toujours dans de nombreux pays d'Afrique et certains pays d'Asie.

Plusieurs enquêtes ethnographiques contemporaines sont ainsi disponibles sur le Népal (Lundström-Baudais et al., 2002), le Sénégal (N’Dove, 1994), l'Asie et l'Afrique (Murty et Kumar, 1995), le monde (ICRISAT et FAO, 1996).

À cela s'ajoutent les enquêtes ethnohistoriques réalisées sur plusieurs provinces françaises et quelques pays européens, rassemblées lors du congrès international sur le millet qui a eu lieu à Aizenay (Vendée) en 1990 (Horandner, 1995).

Les écrits de différents historiens spécialistes des céréales ou de l'alimentation : Adam Maurizio, Jacques André, Georges Comet, ont également été mis à contribution (Maurizio, 1932; André, 1981; Comet, 1992).

$\mathrm{Si}$ les comparatifs ethnologiques sont à utiliser avec discernement, il n'est pas indifférent de noter qu'il existe, selon F. Sigaut, une vraie spécificité du millet dans les modes opératoires employés. Au-delà des divergences de détail, de grandes similarités existent dans les manières de moissonner, d'égrener, de décortiquer, de l'Afrique jusqu'à l'Europe, dans des environnements physiques, culturels et sociaux pourtant très différents et ce sur des siècles, si ce n'est des millénaires (Sigaut, 1995). Comme le souligne K. Lundström-Baudais, ces convergences découlent vraisemblablement des caractères intrinsèques de la plante. Ceux-ci ne semblent laisser que peu d'amplitude dans le choix des techniques.

\section{Le décorticage}

Cette opération semble aller tellement de soi, pour le millet commun en tout cas, qu'elle n'est pas toujours décrite, parfois même à peine mentionnée. Il faut parcourir souvent plusieurs chapitres avant de trouver, au détour d'une page, la locution qui renseigne, telle que : « avant de le cuire il faut évidemment le monder» (Comet 1992, p. 276), ou : «les petits millets comme le millet commun doivent être décortiqués avant d'être consommés " (Murty et Kumar 1995, p. 191). Quoiqu'il en soit, dans tous les ouvrages consultés, la consommation de millet commun vêtu n'est jamais explicitement indiquée. Toutes les enquêtes montrent le décorticage comme une étape obligatoire de la chaîne opératoire, sans même poser la question de sa nécessité. Pour résumer les procédés utilisés, on retrouve tout autour de la planète deux façons de débarrasser le grain de ses glumelles, une directe - le décorticage stricto-sensu - et une indirecte - par broyage -, aboutissant à deux types de produits différents. Le décorticage se fait au moyen d'un pilon et d'un mortier; le produit fini se compose de grains entiers décortiqués et de fragments de glumelles (ces dernières sont ensuite éliminées par vannage). Le second traitement consiste à concasser ou moudre les grains, à l'aide d'une meule, d'un pilon et d'un mortier ou plus récemment d'un moulin mécanique ou électrique. On obtient alors tous les stades intermédiaires entre le gruau grossier et la farine fine, que l'on lave, tamise ou vanne, pour en ôter le son et les restes de glumelles. Dans les deux cas, les enveloppes coriaces ont été éliminées. Les deux procédés se retrouvent indifféremment en Europe, en Afrique, en Asie... et sont souvent employés quelque soit le lieu, en fonction du produit recherché. Pour ne citer que quelques exemples, le décorticage direct par pilage est, ou a été, pratiqué aussi bien au Népal (Lundström-Baudais $e t$ al., 2002), qu'en Inde (Mahias, 1995), au Portugal (Barboff, 1995), en Vendée (Hongrois, 1991) ou en Normandie (Le Cabec et Challavoux, 1995), tandis que le broyage ou mouture des grains suivi d'un lavage ou d'un vannage est décrit également en Vendée (Hongrois, 1995), et dans plusieurs pays d'Afrique et d'Asie (Murty et Kumar, 1995).

Ces opérations longues et fastidieuses nécessitent souvent plusieurs heures de labeur quotidien. Il faut vraiment qu'elles soient incontournables pour que l'on s'y résigne. On comprend toutefois leur nécessité à l'examen des glumelles en question. Celles-ci sont effectivement très épaisses et dures, bien plus coriaces que celles de l'orge dont on a pu tolérer les fragments dans les farines (Procopiou, 2003). Elles sont amères, ne se digèrent pas et ne se transforment pas à la cuisson; même les oiseaux et la volaille les décortiquent avant de les ingérer. À Villiers-sur-Seine à la transition Bronze/Fer, des restes surnuméraires de bases d'épillet de blé et la présence de meules en abondance prouvent, s'il en était besoin, que l'on décortique et broie le blé. Il semble peu plausible qu'on accepte alors de consommer de manière courante le millet avec ses indigestes glumelles. 
Bien entendu il faut rester prudent : plusieurs exemples archéologiques montrent que l'aspect de la nourriture consommée à l'âge du Bronze peut nous sembler parfois très grossier. Il en va ainsi du contenu des pots cités plus haut, ou de certains pains dont la farine est constituée de grains concassés plutôt que véritablement moulus (pains de Corcellettes cités par Maurizio, 1932 et Jacquat, 1989). Différentes études ont en outre établi clairement l'ingestion de millet commun vêtu, par des humains des âges des métaux. L'analyse de l'estomac d'un jeune garçon de l'âge du Fer, tué de coups de poignard et retrouvé ligoté dans les tourbières de Kayhausen en Allemagne a livré un certain nombre de grains et glumelles de millet commun (Behre, 2008) tout comme les coprolithes des travailleurs de la mine de sel de Dürrnberg-bei-Hallen en Autriche (Boencke, 2003). Ces études ne paraissent cependant pas totalement comparables au cas analysé ici. D'une part, elles traitent de situations très particulières, d'autre part il a été démontré qu'après le décorticage il reste toujours une part de grains vêtus (environ 2 à $5 \%$ du total des semences selon Lundström-Baudais et al., 2002). Ceux-ci, non digérés, se retrouvent dans les coprolithes ou les latrines, au contraire des grains nus dont l'amidon a été transformé lors de la cuisson et de la digestion.

\section{Les traitements thermiques}

Comme pour le décorticage, et malgré l'existence de plusieurs variantes, on peut résumer assez simplement les pratiques qui supposent le passage au feu de grains de millets non décortiqués. Un procédé très répandu consiste à chauffer le grain sec sur feu doux ou dans un four. Son objectif peut être de sécher le grain avant de le stocker (Maurizio, 1932, p. 298) ou de le griller avant de le moudre, mais il est surtout employé pour faciliter le décorticage. Cette opération appelée grâlage en Vendée (Hongrois, 1991) a été observée aussi dans le Morbihan, au Portugal, en Autriche ou en Inde et dans bien d'autres régions du monde (Le Cabec et Challavoux, 1995; Barboff, 1995; Gamerith, 1995; Murty et Kumar, 1995; ICRISAT and FAO, 1996). Elle est pratiquée au moins depuis l'Antiquité, puisque Pline (Histoire naturelle L. XVIII, X) précise à propos du millet que cette céréale ne peut être nettoyée sans un passage préalable au feu. Une variante de ce procédé, attestée au Népal consiste à humidifier très légèrement le millet avant de le chauffer. Enfin, bien que la fabrication de la bière de millet ne soit pas décrite en détail dans les sources précédemment citées, son usage est bien connu dans différents pays d'Afrique et d'Asie. Or, le touraillage, étape du maltage qui consiste à stopper la germination par séchage du grain, suppose aussi l'usage du feu sur des grains vêtus.

\section{Expérimentations}

Plusieurs expérimentations ont été faites en juillet 2008 pour reproduire ces traitements thermiques et simuler à leur suite une carbonisation accidentelle. Le maltage a pour l'instant été écarté, car il est peu probable qu'il concerne notre matériel. Il implique en effet que les grains aient germé, ce qui n’a pas été observé sur les blocs de Villiers.

Une expérience supplémentaire a par contre été rajoutée, celle de la confection d'une bouillie de millet. La cuisson des grains dans un liquide (eau, lait ou bouillon) est un mode de préparation très courant, rapporté dans toutes les études ethnographiques. Sur grains entiers, c'est même à peu près le seul mode de préparation possible. Nous l'avons réalisée à partir de grains vêtus, et non de grains décortiqués comme cela est normalement pratiqué, car nous voulions reproduire exactement la composition des blocs de Villiers.

Le millet commun a été obtenu auprès d'un producteur en agriculture biologique installé en Picardie ${ }^{1}$. Il provient de la récolte 2007, qui est passée par un séchoir à soufflerie avant d'être stockée (la panicule de millet qui ne mûrit pas de manière homogène est cueillie partiellement verte, avant que la partie mature ne s'égrène. Pour éviter une humidité excessive qui serait nuisible à une bonne conservation, il faut donc faire sécher la récolte avant de l'entreposer).

\section{Les expériences}

Une série d'expérience a été menée sur trois types de préparations distinctes :

- 200 grammes de millet commun vêtu, sans adjonction de liquide;

- 200 grammes de millet humidifié avec un volume d'eau égal à $5 \%$ du volume de céréale;

- 200 grammes de millet avec 2,5 fois son volume en eau.

Les expériences ont été conduites sur un feu de bois, dans une céramique de cuisson de $20 \mathrm{~cm}$ de diamètre, posée sur une grille à environ $10 \mathrm{~cm}$ au-dessus du feu. Elles ont été répétées plusieurs fois, avec et sans couvercle, sur un feu maintenu le plus possible rougeoyant et sans flammes. Les préparations ont été laissées sur le feu jusqu'à carbonisation complète (figures 5 et 6 ).

Il est bien évident que la température expérimentée ici est plus élevée que la température normale des traitements thermiques, mais elle est supposée être celle d'une carbonisation accidentelle. Il est tout aussi évident que l'expérimentation n'a pas fait le tour de toutes les situations possibles, notamment en termes de quantités de grains.

1. Olivier Delorme, 3 rue du Château, Hameau de Droizelles, 60440 Vercigny. 


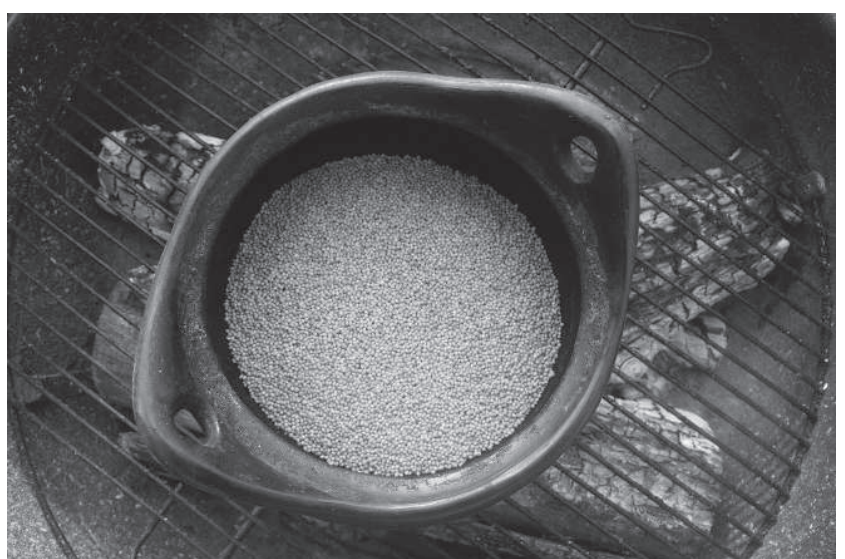

Figure 5 : Expérimentation de carbonisation de millet commun sans ajout d'eau : 1- En début de cuisson (cliché : F. Toulemonde). Figure 5: Charring experiment of broomcorn millet without water addition: 1-At the beginning of the experiment (picture: F. Toulemonde).

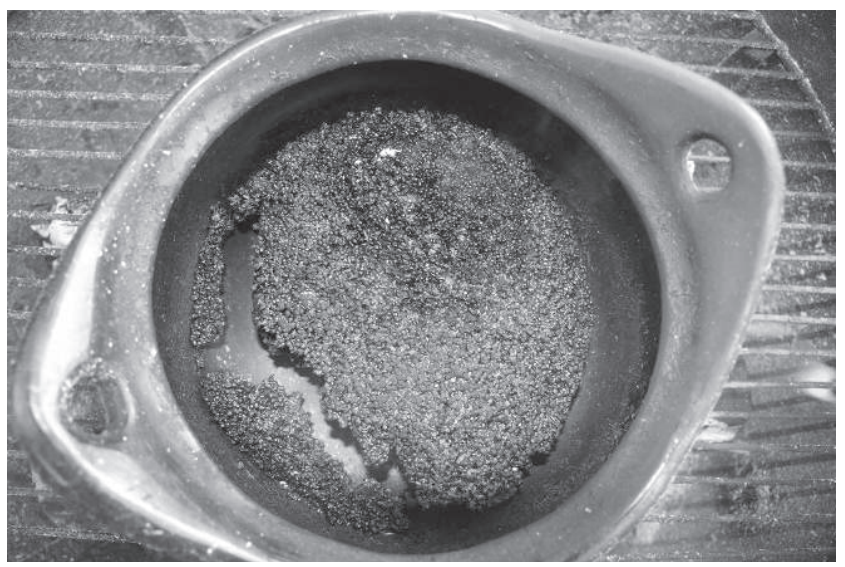

Figure 6 : Expérimentation de carbonisation de millet commun sans ajout d'eau : 2-Après 1 heure de cuisson (cliché : F. Toulemonde).

Figure 6: Charring experiment of broomcorn millet without water addition: 2-After 1 hour of cooking (picture: F. Toulemonde).

\section{Observations}

L'expérimentation a permis quelques observations très intéressantes. On note tout d'abord qu'il n'est pas besoin d'humidité ni d'une forte chaleur, du type de celle que peut générer un incendie, pour que les grains de millet s'agglutinent à la cuisson. La carbonisation à feu modéré sur un feu de bois (donc tout de même quelques centaines de degrés) suffit pour que des grains, même secs, forment un bloc, au bout d'un temps qui varie, pour les deux premiers types de préparation, entre $30 \mathrm{~min}$. et $1 \mathrm{~h} 30$. On remarque ensuite qu'il n'y a pas de différence notable entre les grains secs et les grains légèrement humidifiés : les deux ensembles forment, après carbonisation, des blocs compacts de graines, très proches de ceux découverts à Villiers-sur-Seine. Enfin, l'ajout d'eau en quantité ne favorise pas l'agglutination, bien au contraire. La carbonisation prend alors environ deux fois plus de temps (entre $1 \mathrm{~h} 30$ et 3 heures pour le troisième type de préparation), et les conglomérats sont beaucoup moins compacts. Examinés sous la loupe binoculaire, les blocs présentent de nombreux espaces vides entre les grains, qui ne sont parfois reliés que par quelques points de contact et non toute la surface des glumelles (figure 7). L'eau, en bouillonnant, a produit une masse de millet très aérée qui s'est figée ainsi lors de la carbonisation. De ce fait, les conglomérats sont très friables et se désagrègent facilement au toucher, ce qui n'est pas du tout le cas des blocs archéologiques de Villiers.

\section{Discussions ET CONCLUSIONS}

À l'interrogation initiale : "Quelle interprétation donner aux conglomérats de millet commun découverts à Villierssur-Seine à la transition Bronze/Fer? Peut-on parler de préparation alimentaire? ", les résultats de l'enquête présentée ici fournissent quelques éléments de réflexion, même s'ils n'apportent pas de réponse définitive. On s'aperçoit que la consommation de grains de millet non décortiqués, sous quelque forme que ce soit, n'est pas indiquée explicitement dans les références ethnographiques et ethnohistoriques, pourtant nombreuses et détaillées. L'élimination des glumelles de millet, particulièrement dures et difficiles à assimiler, semble être la norme, et apparaît vraisemblable à une époque où on décortique couramment d'autres céréales. Les préparations alimentaires du genre bouillie de grains, qui viennent spontanément à l'esprit quand on examine ce type de reste carpologique, sont peu à même, si on en croit l'expérimentation, d'en représenter l'état initial. Elles ne sont pas propices à des carbonisations accidentelles récurrentes car elles sont longues à brûler. Elles donnent en outre des agglomérats peu compacts, friables et de conservation aléatoire. L'enquête et les expérimentations ont par contre mis en évidence que certains traitements thermiques, universellement connus et utilisés, en particulier pour faciliter le décorticage, pouvaient produire des restes semblables à ceux soumis à l'étude, dans un intervalle de temps bien plus court. Si les conclusions semblent donc converger plutôt vers ce second type d'interprétation, il faut cependant rappeler qu'il existe dans la documentation archéologique des exemples allant à l'encontre de la théorie d'un décorticage systématique (exemples qu'il conviendrait d'analyser plus 


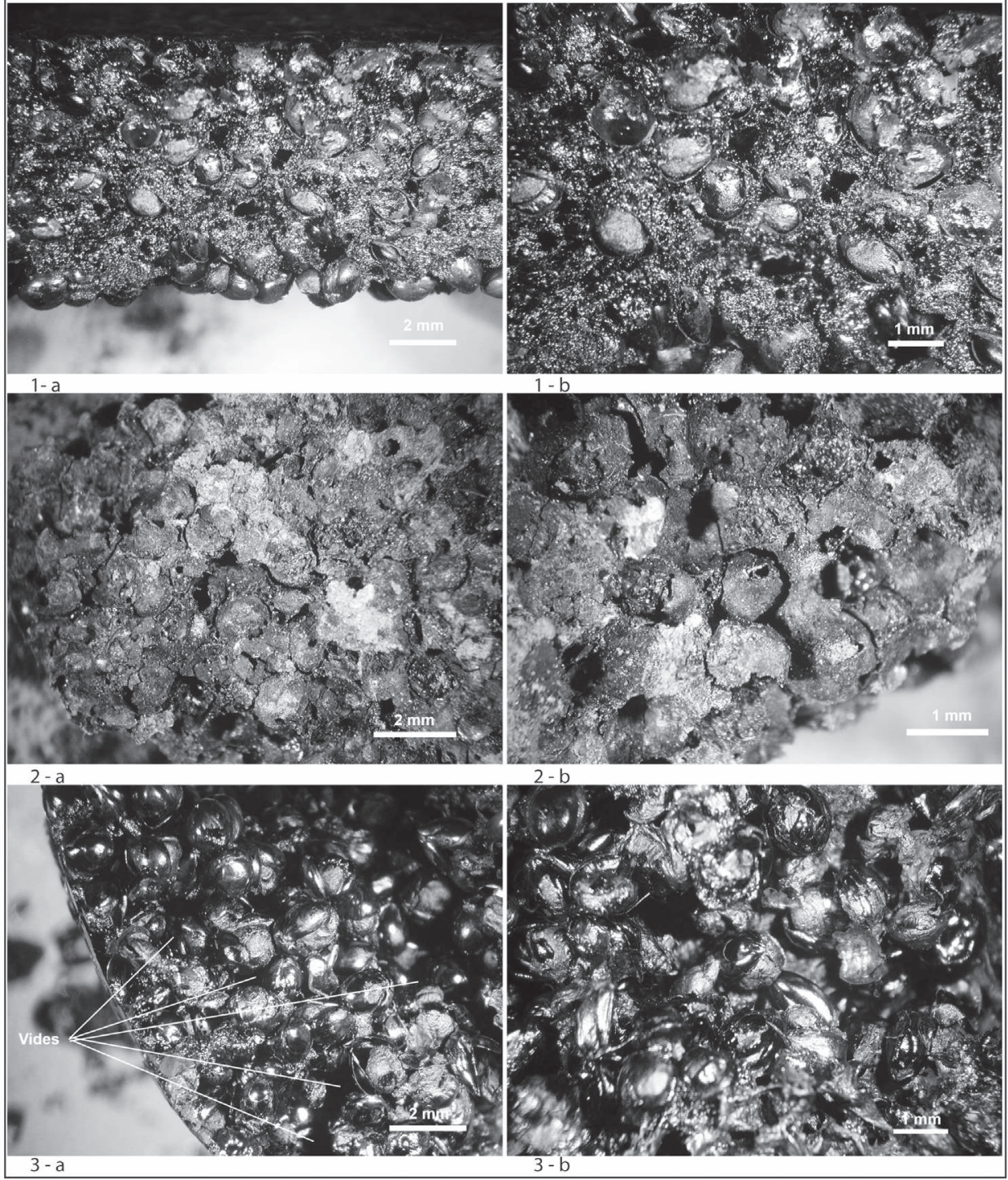

Figure 7 : Résultat des expérimentations : vue sous loupe binoculaire de millet carbonisé à sec (1-a et 1-b) et de millet carbonisé dans de l'eau (3-a et 3-b), à rapprocher des blocs archéologiques de millet de Villiers (2-a et 2-b).

Figure 7: Result of experiments: binocular view of millet charred without water addition (1-a and $1-b)$ and of millet charred with water addition (3-a and 3-b), to compare with archaeological millet from Villiers (2- $a$ and 2-b). longuement). Par ailleurs, les enquêtes ethnographiques et les expérimentations ont leurs limites et ne peuvent répondre qu'aux questions qu'on leur pose. Au-delà d'une préparation alimentaire ou d'un traitement thermique, peut-être existe$\mathrm{t}$-il d'autres explications quant à la présence de ces blocs de millet, hors de tout lien avec une quelconque chaîne opératoire? L'habitat de Villiers-sur-Seine est exceptionnel, on y trouve un nombre important de fosses détritiques, contenant de grandes quantités de restes de faune, attestant de choix opérés dans la consommation carnée et suggérant la tenue de repas collectifs (Auxiette et al., à paraître, Peake et al., à paraître). La découverte sur toute la surface du site de ces nombreux restes agglutinés de millet pourrait renvoyer aussi à certaines activités particulières telles que fêtes (fête du millet?) et festins. L'hypothèse d'offrandes rituelles doit être également évoquée. Ce dernier domaine d'interprétation n'est pas sans référent historique : J. André cite les Fornacalia, fête romaine de la torréfaction du blé où l'on faisait des offrandes de blé grillé; R. Bonnain parle d'offrandes aux morts de gâteaux de millet "pur ", c'est-à-dire mondé, lors des fêtes de la Toussaint en Aquitaine (André, 1981 ; Bonnain, 1995). À Villiers-sur-Seine, la découverte des blocs de millet dans des dépotoirs domestiques favorise cependant peu ce genre d'interprétation.

\section{Remerciements}

Le travail présenté ici a été réalisé dans le cadre d'une thèse de doctorat de l'Université de Paris 1. Cette thèse a bénéficié d'une allocation de recherche de la direction de l'architec- 
ture et du patrimoine du ministère de la Culture et de la Communication. Elle s'est déroulée au sein du laboratoire d'archéobotanique de l'UMR 7209 du CNRS/MNHN, et a pu être menée à son terme grâce à la mise à disposition des locaux et des outils de travail du laboratoire, et au soutien de toute son équipe, notamment de Stéphanie Thiébault et de Véronique Matterne, respectivement directrice et tutrice de thèse.

\section{Bibliographie}

André J., 1981. L'alimentation et la cuisine à Rome, Paris, Les Belles Lettres.

Auxiette G., Peake R., Toulemonde F., à paraître. Food production and diet during the Late Bronze age in the upper Seine Valley (France). In Proceedings of the International Workshop "Socio-Environmental Dynamics over the Last 12,000 Years: The creation of Landscapes III". Session 10: "Setting the Bronze Age Table: Production, Subsistence, Diet and their Implications for European Landscapes”, Kiel 16-18 April 2013.

Bakels C.C., 1983. Les graines carbonisées de Fort-Harrouard (Eure-et-Loir). Antiquités Nationales, 14/15, 1982-83 : 59-63.

Barboff M., 1995. Le millet au Portugal. In E. Horandner (ed.). Millet, actes du Congrès d'Aizenay, 18-19 aồt 1990. Peter Lang, Francfort, 113-121.

BeHre K.-E., 2008. Collected seeds and fruits from herbs as prehistoric food. Vegetation History and Archaeobotany 17: 65-73.

Boencke N., 2003. Organic resources: food supply and raw materials of the miners. In T. Stöllner (ed). The economy of Dürrnbergbei-Hallein: An IronAge salt-mining centre in the Aurstrian Alps. The Antiquaries Journal, 83 : 147-149.

Bonnamour L., Marinval Ph., 1985. Céramiques gallo-romaines précoces avec dépôt de millet, dans la moyenne vallée de la Saône. Revue Archéologique de l'Est et du Centre Est de la France, $36: 321-325$.

Bonnain R., 1995. Le pain des morts. In Horandner E. (ed.). Millet, actes du Congrès d'Aizenay, 18-19 août 1990. Peter Lang, Francfort, 187-196.

Bouby L., Fages G., Treffort J.-M., 2005. Food storage in two Late Bronze Age caves of Southern France: palaeoethnobotanical and social implications. Vegetation History and Archaeobotany 14 : 313-328.

Comet G., 1992. Le paysan et son outil. Essai d'histoire technique des céréales (France VIII'-XVe siècles). Collection de l'école française de Rome 165, Palais Farnèse, Rome.

Gamerit A., 1995. " Hirsch " und "Pfennich ». In Horandner E. (ed.). Millet, actes du Congrès d'Aizenay, 18-19 août 1990. Peter Lang, Francfort, 5-18.
Hongrois C., 1991. Si t'aimes pas l'meuille... : culture et consommation du millet en Vendée, Office Municipal des Affaires Culturelles, Aizenay.

Hongrois M., 1995. Do mell en Vendée ou culture et consommation du mil en Vendée, dans le canton de la Chataigneraie. In E. Horandner (ed.). Millet, actes du Congrès d'Aizenay, 18-19 août 1990. Peter Lang, Francfort, 71-86.

Horandner E. (ed.), 1995. Millet, actes du Congrès d'Aizenay, 18-19 août 1990. Peter Lang, Francfort.

ICRISAT (International Crops Research Institute for the SemiArid Tropics), FAO (Food and Agriculture Organization of the United Nations), 1996. The World Sorghum and Millet Economies: Facts, Trends and Outlook, [http://www.fao.org/ docrep/W1808E/w1808e00.HTM], consulté le 17/11/2009.

JACQUAT C., 1989. Hauterive-Champréveyres 2 : les plantes de l'Âge du Bronze. Contribution à l'histoire de l'environnement et de l'alimentation. Archéologie Neuchateloise 8. Éditions du Ruau, Saint Blaise.

Le Cabec Y., Chavaloux J., 1995. Ferme archéologique de Melrand - Début d'enquête sur le millet à Melrand (Morbihan). In E. Horandner (ed.). Millet, actes du Congrès d'Aizenay, 18-19 août 1990. Peter Lang, Francfort, 97-100.

Lundstrom-Baudais K., Rachoud-Schneider A.-M., Baudais D., Poissonnier B., 2002. Le broyage dans la chaîne de transformation du millet (Panicum miliaceum) : outils, gestes et écofacts. In H. Procopiou, R. Treuil (dir.). Moudre et Broyer, I. Méthodes. CTHS, Paris, 181-209.

Mahias M.-C. 1995. Aspects de la culture et de la consommation du mil en Inde. In E. Horandner (ed.). Millet, actes du Congrès d'Aizenay, 18-19 août 1990. Peter Lang, Francfort, 171-186.

Maurizio A., 1932. Histoire de l'alimentation végétale. Payot, Paris.

Murty D.S., Kumar K.A., 1995. Traditional uses of sorghum and millets. In D.A.V. Dendy (ed). Sorghum and Millets: Chemistry and Technology. American Association of Cereal Chemists Inc., St. Paul, Minnesota, 185-221.

N’Dove A.-S., 1994. La transformation du mil au Sénégal. Industrie des céréales. 88, juillet-aout-septembre 1994 : 23-27.

Peake R., Allenet G., Auxiette G., Boisseau F., Chaussé C., Coubray S., Leroyer C., Pautret-Homerville C., Perrière J., Toulemonde F., 2009. Villiers-sur-Seine, Le Gros Buisson. Un habitat aristocratique de la fin de l'âge du Bronze et du début du premier âge du Fer. In M.-J. RoulièreLambert, A. Daubigney, P.-Y. Milcent, M. Talon, J. Vital (ed.). De l'Âge du Bronze à l'Âge du Fer en France et en Europe occidentale (X'-VII s. av. J.-C.); la moyenne vallée du Rhône aux âges du Fer, Actes du XXX'e colloque international de l'AFEAF, coorganisé avec l'APRAB, Saint-Romain-en-Gal, 26-28 Mai 2006. Dijon, 2009. Revue archéologique de l'Est, suppl. 27, 559-564.

Peake R. (dir.), à paraître. Monographie du site de Villiers-surSeine, "Le Gros Buisson" (Seine-et-Marne, Ile-de-France), un 
habitat aristocratique de la transition âge du Bronzelâge du Fer. Collection "Recherches archéologiques ", co-édition Inrap/ CNRS Éditions.

Procopiou H., 2003. Les techniques de décorticage dans le monde égéen. Étude ethnoarchéologique dans les Cyclades. In Anderson P.C., Cummings L.S., Schippers T. K.

Simonel B. (dir.). Le traitement des récoltes : un regard sur la diversité du Néolithique au présent, Actes des XXIII ${ }^{\mathrm{e}}$ rencontres internationales d'archéologie et d'histoire d'Antibes. APDCA, Antibes, 115-136.

Sigaut F., 1995. Les millets en Eurasie, d'une fête populaire à des questions pour les chercheurs. In E. Horandner (ed.). Millet, actes du Congrès d'Aizenay, 18-19 août 1990. Peter Lang, Francfort, 133-154.

Toulemonde F., 2006. Étude carpologique d'un site de $1^{\text {er }} \hat{\text { Age }} \mathrm{du}$ Fer en Île-de-France, Villiers-sur-Seine Le Gros Buisson. Mémoire de Master 1, Université de Paris I, France.

Toulemonde F., 2010. L'alimentation végétale durant la Protohistoire ancienne en Île-de-France. Étude carpologique des sites de Gif-sur-Yvette "Rond-point de Corbeville » (Essonne) et Villiers-sur-Seine " Le Gros Buisson " (Seine-etMarne). Revue archéologique d'Île-de-France 3 : 63-83. 